Highly Energetic Physical Processes and

Mechanisms for Emission from Astrophysical Plasmas

IAU Symposium, Vol. 195, 2000

P. C. H. Martens, S. Tsuruta, and M. A. Weber, eds.

\title{
Particle Acceleration at Astrophysical Shocks
}

\author{
A. Achterberg ${ }^{1}$ \\ Sterrenkundig Instituut, Postbus 80000, 3508 TA Utrecht, The \\ Netherlands
}

\begin{abstract}
The acceleration of particles in the vicinity of astrophysical shocks has become the main paradigm in astrophysics for the production of superthermal and relativistic particles. In this review, I consider the basic theory of shock acceleration and the open questions.
\end{abstract}

\section{Introduction}

Since Baade \& Zwicky (1934) suggested that galactic cosmic rays are associated with supernovae, astrophysicists have looked for models explaining the production of cosmic ray nucleons. Radio astronomy established the presence of relativistic $(\sim \mathrm{GeV})$ electrons in sources such as supernova remnants and active galaxies from the synchrotron radiation they emit in $|\boldsymbol{B}| \sim 1-10 \mu \mathrm{G}$ magnetic fields. The energy distribution of these electrons is quite similar to the spectrum observed for galactic cosmic rays: a power-law in energy $\mathcal{E}$ :

$$
N(\mathcal{E}) \mathrm{d} \mathcal{E} \propto \mathcal{E}^{-s} \mathrm{~d} \mathcal{E}, \quad \text { with } \quad s \sim 2.5-2.7,
$$

suggesting a common production process. The observation of synchrotron emission of X-rays by $10-100 \mathrm{TeV}$ electrons in SN1006 (Koyama et al. 1995) shows that the largest energies observed for galactic cosmic ray nucleons can indeed be achieved in SNRs by electrons of similar rigidity $\mathcal{R} \sim \mathcal{E} / q$.

Most theoretical effort until 1977 was devoted to [1] identifying the agent responsible for the acceleration and [2] explaining the narrow range of values for the power-law index $s$ observed in a variety of objects.

Fermi (1949), in a seminal paper, proposed distributed acceleration of cosmic rays in the magnetised interstellar medium (ISM) as a result of the interaction with turbulent motions ("clouds"). Parker (1965) suggested hydromagnetic waves as the acceleration agent. In stochastic Fermi acceleration, particles diffuse in energy due to random momentum changes induced by the waves (see, e.g., Hall \& Sturrock 1967). The slope $s$ of the energy distribution is determined by the competition between the energy gain and the particle losses from the acceleration region (Fermi 1949). If the particle energy increases with time as $\mathcal{E}(t)=\mathcal{E}_{0} e^{t / \tau}$, and the survival probability of a particle in the acceleration region decays as $\mathcal{P}_{\text {surv }}=e^{-t / T}$, the slope is determined by the ratio of characteristic

\footnotetext{
${ }^{1}$ Also at: Center for High Energy Astrophysics, Amsterdam, The Netherlands
} 
times $\tau / T$ :

$$
s=1+\frac{\tau}{T} .
$$

The "nearly universal" values for $s$ require a feedback mechanism constraining the acceleration and escape times to $\tau \sim T$ (Burn 1975; Achterberg 1979).

Interactions between hydromagnetic waves in the ISM and cosmic rays confine the bulk of the cosmic rays within the galaxy for some $10^{7}$ years. Cosmic ray streaming leads to the (resonant) generation of Alfvén waves with a wavelength comparable with the gyration radius: $\lambda \sim r_{\mathrm{g}} \sim p c / q B$ (Kennel \& Petchek 1966; Wentzel 1974). Here, $p$ is the particle momentum, $q$ is the particle charge, and $B$ is the strength of the magnetic field. Wave-particle scattering limits the mean free path along the field to

$$
\ell_{\mathrm{mfp}} \sim r_{\mathrm{g}} / \mathcal{I}\left(k \sim r_{\mathrm{g}}^{-1}\right), \quad B_{\mathrm{rms}}^{2}=B^{2} \int \mathcal{I}(k) \mathrm{d}[\ln k] .
$$

Here, $\mathcal{I}(k)$ is the relative intensity of the magnetic fluctuations of rms amplitude $B_{\mathrm{rms}}$ at $k=2 \pi / \lambda$. This self-confinement couples cosmic rays to the fluid motions (Parker 1965; Skilling 1975a, b). This coupling is an important ingredient of shock acceleration theory. For a review, see Dolginov \& Katz (1994).

\section{Shocks as Particle Accelerators}

Colgate and Johnson (1966) were the first to consider the supernovae blast wave as a direct accelerating agent. In their model, the mechanical energy $\left(\sim 10^{51} \mathrm{erg}\right)$ of the supernova explosion is imparted preferentially to the tenuous outer layers of the imploding star, raising the energy per particle of this material far above the mean. Shatzman (1963) proposed a model where particles interact with an oblique shock (upstream magnetic field $\boldsymbol{B}_{\mathrm{u}}$ not along the shock normal $\hat{n}$ ), and predicted that this should lead to a power-law energy spectrum.

This process, now known as Shock Drift Acceleration (SDA), involves a single interaction event between particle and shock. A particle drifts in the direction of the flow-induced electric field, $\boldsymbol{E}=-V_{\mathrm{s}}\left(\hat{\boldsymbol{n}} \times \boldsymbol{B}_{\mathrm{u}}\right) / c$ with $V_{\mathrm{s}}$ the shock speed, as a consequence of the magnetic field compression: $\left|\boldsymbol{B}_{\mathrm{d}}\right| \geq\left|\boldsymbol{B}_{\mathrm{u}}\right|$, with $\boldsymbol{B}_{\mathrm{d}}$ the downstream field. The radius of curvature of the gyro-orbit, $r_{\mathrm{g}} \propto$ $|\boldsymbol{B}|^{-1}$, is smaller on the downstream side, leading to a drift along $\boldsymbol{E}$ as long as the particle orbit intersects the shock. For nearly perpendicular shocks (shock normal $\hat{\boldsymbol{n}} \perp \boldsymbol{B}_{\mathrm{u}}$ ), the total energy gain can be interpreted as resulting from the adiabatic invariance of the magnetic moment (e.g., Hudson 1965; Alekseyev \& Kroptokin 1970; Whipple, Northrop, \& Birmingham 1986):

$$
\mathcal{M}=p_{\perp}^{2} / 2|B|=\text { constant. }
$$

Here, $p_{\perp}$ is the component of particle momentum perpendicular to the magnetic field. This conservation law is valid if the shock crossing involves many gyration loops. SDA has been considered by a number of authors; for a review, see Armstrong, Pesses, \& Decker (1985).

Self-confinement of particles by hydromagnetic waves can lead to repeated shock-particle interactions (Axford, Leer, \& Skadron 1977; Krimsky 1977; Bell 
1978; Blandford \& Ostriker 1978). In Diffusive Shock Acceleration (DSA), particles gain energy at shock crossings through scattering between up- and downstream waves: a form of regular Fermi acceleration that also works for parallel shocks where $\boldsymbol{B}_{\mathbf{u}} \| \hat{\boldsymbol{n}}$. Reviews of DSA (Drury 1983; Blandford \& Eichler 1987; Jones \& Ellison 1991; Kirk 1994) contain the technical details that I omit here.

\section{Basic Theory of Nonrelativistic DSA}

Shock acceleration proceeds according to a simple scenario: particles gain energy at shock crossings due to the velocity difference between up- and downstream scattering centers (waves) advected by the flow, but at each cycle, from upstream to downstream and back, some particles are lost downstream. If scattering is frequent, the momentum gain per cycle and escape (return) probability $\mathcal{P}_{\text {esc }}$ $\left(\mathcal{P}_{\text {ret }}\right)$ for a parallel shock with velocity $V_{\mathrm{s}}$ and compression ratio $r$ are (see, e.g., Bell 1978)

$$
\left\langle\frac{p_{n+1}}{p_{n}}\right\rangle=1+\frac{4(r-1) V_{\mathrm{s}}}{3 r v}, \quad \mathcal{P}_{\mathrm{esc}}=1-\mathcal{P}_{\text {ret }}=\frac{4 V_{\mathrm{s}}}{r v},
$$

with $v$ the particle velocity and $n$ the cycle number. Particles can interact repeatedly with the shock if $v \gg V_{\mathrm{s}}$ so that $\mathcal{P}_{\text {esc }} \ll 1$. The competition between momentum gain and escape from the shock leads to a power-law momentum distribution above the injection momentum, $N(p) \mathrm{d} p \propto p^{-s} \mathrm{~d} p$, with slope

$$
s=1+\frac{\ln \left(1 / \mathcal{P}_{\text {ret }}\right)}{\ln \left\langle p_{n+1} / p_{n}\right\rangle}=\frac{r+2}{r-1} .
$$

This slope depends only on the shock compression ratio $r$ : the needed "fine tuning". These results remain valid for oblique shocks with compression ratio $r$, provided one re-interprets $V_{\mathrm{s}}$ as the normal component of the flow velocity with respect to the shock normal (Bell 1978; Drury 1983).

The details of the diffusive particle transport near the shock determine the typical length-, time-, and energy scales of DSA. The balance between diffusion away from the shock and advection into the shock leads to an exponential upstream precursor on a diffusion scale, $L_{\text {diff }} \sim \kappa_{\mathrm{n}} / V_{\mathrm{s}}$, with $\kappa_{\mathrm{n}}=\hat{\boldsymbol{n}} \cdot \boldsymbol{\kappa} \cdot \hat{\boldsymbol{n}}$ the component of the diffusion tensor along the shock normal. The level of scattering also determines the (energy-dependent) cycle time $t_{\text {cy }}$, the acceleration time $t_{\text {acc }}$, and the time $T(\mathcal{E})$ to reach a certain energy $\mathcal{E}$ (see, e.g., Forman \& Morfill 1979; Axford 1981; Drury 1983; 1991):

$$
t_{\mathrm{cy}} \sim \frac{4 \kappa_{\mathrm{n}}}{v V_{\mathrm{s}}}, \quad t_{\mathrm{acc}} \equiv \frac{t_{\mathrm{cy}}}{(\Delta p / p)} \sim \frac{4 \kappa_{\mathrm{n}}}{V_{\mathrm{s}}^{2}}, \quad T(\mathcal{E})=\int_{\mathcal{E}_{\mathrm{inj}}}^{\mathcal{E}} t_{\mathrm{acc}}\left(\mathcal{E}^{\prime}\right) \frac{\mathrm{d} \mathcal{E}^{\prime}}{\mathcal{E}^{\prime}}
$$

\subsection{Maximum Energy}

Diffusive shock acceleration only produces particles up to an energy $\mathcal{E}_{\max }$ where the net energy gain per cycle equals the energy losses in a cycle time. If losses are unimportant, the finite age $t_{\mathrm{s}}$ or finite size $R_{\mathrm{s}}$ of the source limits the energy. The most stringent of the three conditions

$$
t_{\text {acc }}\left(\mathcal{E}_{\max }\right) \sim t_{\text {loss }}\left(\mathcal{E}_{\max }\right), \quad T\left(\mathcal{E}_{\max }\right) \sim t_{\mathrm{s}}, \quad \text { or } \quad L_{\text {diff }}=\kappa_{\mathrm{n}}\left(\mathcal{E}_{\max }\right) / V_{\mathrm{s}} \sim R_{\mathrm{s}}
$$


determines the maximum energy $\mathcal{E}_{\max }$. Here, $t_{\text {loss }} \equiv|\mathrm{d}(\ln \mathcal{E}) / \mathrm{d} t|_{\text {loss }}^{-1}$.

One often assumes that the mean free path roughly equals the particle gyration radius, $\ell_{\mathrm{mfp}} \sim r_{\mathrm{g}}(\mathcal{E}) \sim \mathcal{E} / q|B|$, for relativistic particles. The associated Bohm diffusion coefficient is $\kappa_{\mathrm{B}}=c r_{\mathrm{g}}(\mathcal{E}) / 3 \propto \mathcal{E}$, which corresponds to strong

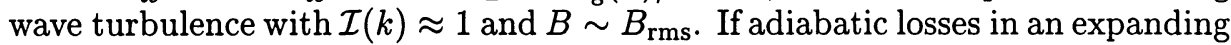
shock wave $\left(t_{\text {loss }} \sim R_{\mathrm{s}} / V_{\mathrm{s}}\right)$ or the shock size/age limits $\mathcal{E}_{\max }$, one has for a particle with charge $q=Z e$ undergoing Bohm diffusion:

$$
\mathcal{E}_{\max }=Z e B \beta_{\mathrm{s}} R_{\mathrm{s}}
$$

This is an absolute upper limit for the energy produced by nonrelativistic DSA.

Table 1. Maximum energy of particles produced by DSA.

\begin{tabular}{|c|c|c|c|c|c|}
\hline Object & $R_{\mathrm{s}}$ & $\beta_{\mathrm{s}}$ & $B(\mathrm{G})$ & limiting mechanism & $\mathcal{E}_{\max }(\mathrm{eV})$ \\
\hline $\begin{array}{l}\text { Solar Flare } \\
\text { Type II shock }\end{array}$ & $10^{10} \mathrm{~cm}$ & $10^{-2.5}$ & 10 & $\begin{array}{l}\text { protons: size/age } \\
\text { electrons: synchr. losses }\end{array}$ & $\begin{array}{l}10^{11} \\
10^{9}\end{array}$ \\
\hline $\begin{array}{l}\text { Interplanetary } \\
\text { Shocks }\end{array}$ & $10^{13} \mathrm{~cm}$ & $10^{-3}$ & $10^{-5}$ & $\begin{array}{l}\text { protons: } \text { size/age } \\
\text { electrons: size/age }\end{array}$ & $\begin{array}{l}10^{8} \\
10^{8}\end{array}$ \\
\hline $\begin{array}{l}\text { Supernova } \\
\text { Remnants }\end{array}$ & $10 \mathrm{pc}$ & $10^{-2.5}$ & $10^{-4}$ & $\begin{array}{l}\text { protons: size/age } \\
\text { electrons: synchr. losses }\end{array}$ & $\begin{array}{l}10^{15} \\
10^{12}\end{array}$ \\
\hline $\begin{array}{l}\text { Gamma Ray } \\
\text { Bursts }\end{array}$ & $0.01 \mathrm{pc}$ & 1 & $10^{-6}$ & $\begin{array}{l}\text { protons: size/age } \\
\text { electrons: } \text { size/age }\end{array}$ & $\begin{array}{l}10^{15} \\
10^{15}\end{array}$ \\
\hline AGN & $10^{13} \mathrm{~cm}$ & 0.1 & $10^{4}(?)$ & $\begin{array}{l}\text { protons: pion losses } \\
\text { electrons: SC losses }\end{array}$ & $\begin{array}{l}10^{16} \\
10^{11}\end{array}$ \\
\hline $\begin{array}{l}\text { Hot Spots } \\
\text { radio galaxy }\end{array}$ & $10 \mathrm{kpc}$ & $\sim 1$ & $10^{-4}$ & $\begin{array}{l}\text { protons: shock size } \\
\text { electrons: synchr. losses }\end{array}$ & $\begin{array}{l}10^{20} \\
10^{14}\end{array}$ \\
\hline $\begin{array}{l}\text { Cosmic Large } \\
\text { Scale Structure }\end{array}$ & $10 \mathrm{Mpc}$ & $10^{-2.5}$ & $10^{-6}$ & $\begin{array}{l}\text { protons: pion losses } \\
\text { electrons: SC losses }\end{array}$ & $\begin{array}{l}10^{19} \\
10^{14}\end{array}$ \\
\hline
\end{tabular}

Table 1 shows typical values for $\mathcal{E}_{\max }$ in a number of objects. These values have been calculated for adiabatic losses in expanding shock waves, for pion production losses (for constant loss length $\ell_{\pi}$ ) suffered by highly-relativistic protons (and neutrons) due to collisions with ambient nuclei $(N)$ or photons $(\gamma)$ in reactions like $p+\gamma \longrightarrow p+\pi$ or $p+N \longrightarrow p+N+\pi$, and for the well-known synchro-Compton losses (loss length $\ell_{\text {loss }}(\mathcal{E})=\ell_{\text {sc }}\left(m c^{2} / \mathcal{E}\right)$ ) of relativistic electrons (or ultra-relativistic protons in AGN) due to the ambient magnetic/radiation field. Pion production losses occur in Active Galactic Nuclei where the radiation density is very high (see, e.g., Zdziarski 1986; Kazanas \& Ellison 1986; Sikora et al. 1987; and Begelman, Rudak, \& Sikora 1990), and limits the production energy of the Ultra High Energy Cosmic Rays $\left(\mathcal{E} \geq 10^{18.5} \mathrm{eV}\right)$ (see, e.g., Norman, Melrose, \& Achterberg 1995). From this table, one sees that, except for extreme (and rare!) situations associated with powerful radio galaxies and Large Scale Structure, protons are never accelerated much beyond $\sim 10^{15} \mathrm{eV}$, nor are electrons much beyond $\sim 1 \mathrm{TeV}$ : a sort of "cosmic conspiracy". 


\section{Nonlinear Shock Acceleration}

Generally, the upstream flow is decelerated by the pressure gradient associated with the particle precursor, communicated to the flow by the scattering waves (Achterberg 1981). This introduces a nonlinearity with important consequences. [1] It decreases the Mach number and the compression ratio $r_{\mathrm{s}}$ of the shock. In cosmic-ray-dominated "shocks", the true gas shock (subshock) disappears altogether so that the velocity transition becomes smooth on a scale of order the cosmic ray diffusion scale $L_{\text {diff }} \sim \kappa / V_{\mathrm{s}}$. [2] It can increase the overall compression $r_{\mathrm{t}}$ across the (sub)shock and the precursor region above the maximum value $r=4$ allowed for an ideal, nonrelativistic gas. A large cosmic ray pressure "softens" the equation of state of the mixture. One can have $4 \leq r_{\mathrm{t}} \leq 7$, depending on the pressure ratio $P_{\mathrm{cr}} / P_{\text {gas }}$ in the mixture and the effective specific heat ratio $4 / 3 \leq \tilde{\gamma}_{\mathrm{cr}} \leq 5 / 3$ of the accelerated particles.

If the diffusion length $\kappa_{\mathrm{n}} / V$ increases with energy, high-energy particles diffuse further ahead of the shock and "sample" a larger total compression (Eichler 1984). The spectral slope decreases with energy: a concave spectrum with

$$
\frac{r_{\mathrm{t}}+1}{r_{\mathrm{t}}-1} \leq\left|\frac{\partial \ln N(\mathcal{E})}{\partial \ln \mathcal{E}}\right| \leq \frac{r_{\mathrm{s}}+1}{r_{\mathrm{s}}-1}
$$

with $r_{\mathrm{s}}$ the compression ratio of the subshock.

Self-consistent solutions describing the precursor flow and subshock, as well as the particle momentum distribution, are difficult to find. A simplified theory, proposed by Axford et al. (1977), uses a two-fluid description. The cosmic rays are described in terms of macroscopic quantities: their pressure and energy density (see, e.g., Drury \& Völk 1981; Axford et al. 1982; Achterberg, Blandford, \& Periwal 1984; Duffy, Drury, \& Völk 1994; Drury, Völk, \& Berezhko 1995). These models solve the algebraic equations for energy-momentum conservation in the gas/cosmic-ray mixture, supplemented at the subshock by the RankineHugoniot relations connecting the up- and downstream fluid states. One can find up to three steady-state solutions, including a "high-efficiency" solution where most of the upstream momentum flux is converted into cosmic rays.

The general conclusions based on two-fluid models (high-efficiency solutions; spectral curvature) have been confirmed by numerical calculations, ranging from the full solutions of the hydrodynamic equations and coupled cosmic ray transport equations (e.g., Falle \& Giddings 1987) to Monte-Carlo methods where the interaction between cosmic rays and waves is modelled by some scattering law (e.g., Ellison et al. 1993; Baring, Ellison, \& Jones 1994; Kang \& Jones 1995; Ellison, Baring, \& Jones 1995). The approach has some problems: the value of $\gamma_{\mathrm{cr}}$ is not self-consistent but must be specified; particle injection is not included naturally; and, for flat spectra, the cosmic-ray pressure and effective diffusion coefficient which determines the cosmic ray energy flux are dominated by the time-dependent spectrum near $\mathcal{E}_{\max }$. Therefore, Achterberg et al. (1984) conclude that two-fluid models only apply if the underlying particle distribution has a slope $2<s<3$. Malkov \& Völk (1996) have formulated a "renormalized" theory of cosmic-ray modified shocks which addresses some of these problems by using approximate solutions for the underlying microscopic distribution. 


\section{Injection}

The injection of particles into DSA is probably intimately connected to the detailed (microscopic) processes in the shock. Some models circumvent the details of the shock structure, assuming that some shock-heated downstream particles (ions) leak back upstream, providing the injection at the shock (e.g., Eichler 1979; Edminston, Kennel, \& Eichler 1982). This hypothesis has been reinvestigated by Malkov \& Völk (1995). They develop a formalism for describing interactions between shock-heated particles and self-generated waves at high anisotropies-in essence, a low-energy extension of standard DSA for $v \gtrsim V_{\mathbf{s}}$. They find that a significant fraction of the downstream ions are injected into the acceleration process.

Kang \& Jones (1990) have modelled injection in a two-fluid approach by including the energy of the injected particles as a jump in the cosmic ray energy flux at the subshock. This introduces an additional closure problem: the relation between the energy injection rate at the subshock and the shock parameters. Kang \& Jones find that the postshock cosmic ray pressure is rather insensitive to the details of the injection process.

Direct (hybrid) simulations of collisionless shocks simulate ion orbits but treat the electrons as a fluid. This approach has been reviewed by Scholer, Kucharek, \& Trattner (1998). They conclude that ion injection is mostly the result of the interaction between particles gyrating near the shock front and electric fields along the shock front produced by shock-generated plasma waves, or by waves advected into the shock from upstream.

\section{Stability: Precursor and Shock Stability}

The wave-mediated coupling between cosmic rays and fluid in the precursor may lead to instabilities. The simplest version occurs in a quasi-parallel shock, as pointed out by Drury \& Falle (1986). This so-called squeezing instability results from the modulation of the cosmic ray diffusion, induced by density perturbations. This work was extended by Zank, Axford, \& McKenzie (1990) and by Begelman \& Zweibel (1994). The latter authors include the effect of drift between cosmic rays and fluid, which is destabilizing. They suggest that these instabilities lead to the formation of weak shocks in the precursor. How this influences cosmic ray acceleration has not been investigated in any detail. Mond \& Drury (1998) have considered a different kind of stability: the stability of cosmic-ray-modified shocks against the corrugational instability and the emission of sound waves. They find that the earlier conjecture, that one of the three solutions in the two-fluid approximation must be unstable, is in fact correct.

\section{Inclination Angle Dependence and Quasi-perpendicular Shocks}

The discussion so far has been mostly confined to quasi-parallel shocks with $\theta_{B n} \equiv \cos ^{-1}(\hat{\boldsymbol{n}} \cdot \boldsymbol{B} /|\boldsymbol{B}|) \lesssim \pi / 2$. In quasi-perpendicular shocks, with $\theta_{B n}>\pi / 2$, there are a number of effects which modify the simple picture.

The intersection point of a planar shock and a given fieldline has a velocity $V_{\mathrm{i}}=V_{\mathrm{s}} / \cos \left(\theta_{\mathrm{Bn}}\right)$. Particles constrained to move along field lines can only cross 
the shock into the upstream medium if $(\boldsymbol{v} \cdot \boldsymbol{B}) /|\boldsymbol{B}|>V_{\mathrm{i}}$. Injection by thermal leakage of downstream particles therefore decreases with increasing $\theta_{B n}$. MonteCarlo simulations (Baring, Ellison, \& Jones 1993; 1994; 1995) show that, for high Mach numbers, injection by thermal leakage drops strongly for $\theta_{B n}>30^{\circ}$.

Cosmic ray transport changes qualitatively in quasi-perpendicular shocks: diffusion across the magnetic field becomes important. Diffusion along the shock normal is governed by $\kappa_{\mathrm{n}}=\kappa_{\|} \cos ^{2}\left(\theta_{B n}\right)+\kappa_{\perp} \sin ^{2}\left(\theta_{B n}\right)$, in terms of the fieldaligned and perpendicular diffusion coefficients $\kappa_{\|}$and $\kappa_{\perp}$. For weak collisionality $\left(\ell_{\mathrm{mfp}} \gg r_{\mathrm{g}}\right)$ and nearly perpendicular shocks, the value of $\kappa_{\mathrm{n}} \sim \kappa_{\perp} \approx$ $\kappa_{\mathrm{B}}\left(r_{\mathrm{g}} / \ell_{\mathrm{mfp}}\right)$ is much less than the Bohm value. Since the acceleration time scales as $t_{\text {acc }} \propto \kappa_{\mathrm{n}}$, acceleration proceeds more rapidly - a point made most forcefully by Jokipii $(1982,1987)$. However, this does not necessarily change the maximum energy $\mathcal{E}_{\max }$ that can be achieved. The energy gain at very oblique shocks results from the particle drift along the shock face. For a shock of size $R_{\mathrm{s}}$, the maximum possible energy in the absence of losses is $\mathcal{E}_{\max } \sim Z e B \beta_{\mathrm{s}} R_{\mathrm{s}}$, the same value as derived for quasi-parallel shocks with Bohm diffusion.

\section{Superluminal Shocks and Anomalous Transport}

Oblique shocks with $V_{\mathrm{i}}>c$ are superluminal. A Lorentz boost along the shock face with a velocity $\bar{V}_{\mathrm{HT}}=c /\left[\Gamma_{\mathrm{s}} \beta_{\mathrm{s}} \tan \left(\theta_{B n}\right)\right]$ (de Hoffmann \& Teller 1950) leads to a situation with (crossed) electric and magnetic fields along the shock face, so a straight field line crosses a planar shock instantly. In this case, rapid crossfield diffusion, or some anomalous transport process such as field line wandering (Ball \& Kirk 1992; Achterberg \& Ball 1994) is essential for DSA. In the absence of such processes, only Shock Drift Acceleration can occur.

Models have been proposed for shock acceleration near quasi-perpendicular shocks where the transport of accelerated particles in the immediate vicinity of the shock is anomalous, i.e. not straightforward diffusion due to the scattering action of self-generated waves. Anomalous transport is characterised by nonstandard time behaviour of the dispersion in position $x:\left\langle\left\langle(\Delta x)^{2}\right\rangle\right\rangle \propto t^{\sigma}$, with $\Delta x \equiv x-\langle\langle x\rangle\rangle$. Ordinary diffusion corresponds to $\sigma=1$, the case $\sigma<1$ $(\sigma>1)$ is called subdiffusion (superdiffusion). Most of this work (Achterberg \& Ball 1994; Duffy et al. 1995; Kirk, Duffy, \& Gallant 1996) has concentrated on the transport-caused field line wandering (Jokipii \& Parker 1969; Isichenko 1991).

Low-frequency magnetic perturbations ("magnetic flutter") with relative amplitude $\boldsymbol{h} \equiv \delta \boldsymbol{B}_{\perp} / B_{0}$ in the plane perpendicular to the mean magnetic field $B_{0}$ leads to field line wandering. At a distance $s>L_{\|}$, where $L_{\|}$is the field correlation length along the mean field, the actual field lines diffuse perpendicular to the mean field, the distance $\boldsymbol{r}_{\perp}$ from the mean field scaling as

$$
\left\langle\left\langle\left|\boldsymbol{r}_{\perp}\right|^{2}\right\rangle\right\rangle \sim 2 D_{\mathrm{m}} s, \quad D_{\mathrm{m}} \sim\left\langle\left\langle|\boldsymbol{h}|^{2}\right\rangle\right\rangle L_{\|} .
$$

Here, $D_{\mathrm{m}}$ is a field line diffusion coefficient per unit length. Particles are decorrelated from a patch of field lines after diffusing a distance $L_{\mathrm{dc}} \sim \sqrt{\kappa_{\|} t_{\mathrm{dc}}}$ along the mean field, with $\kappa_{\|} \equiv(\boldsymbol{B} \cdot \boldsymbol{\kappa} \cdot \boldsymbol{B}) / B^{2}$. If $L_{\|}<\ell_{\mathrm{mfp}}<L_{\mathrm{dc}}$, the particles 
diffuse along the field $\left(s \sim \sqrt{2 \kappa_{\|}} t\right)$ and subdiffuse across the field for $t<t_{\mathrm{dc}}$ :

$$
\left\langle\left\langle\Delta x_{\perp}^{2}\right\rangle\right\rangle \sim 2^{3 / 2} D_{\mathrm{m}} \kappa_{\|}^{1 / 2} t^{1 / 2} .
$$

For $t>t_{\mathrm{dc}}$, particles execute ordinary diffusion across the field, with effective perpendicular diffusion coefficient $D_{\perp}=D_{\mathrm{m}}\left(L_{\mathrm{dc}} / t_{\mathrm{dc}}\right)$.

The momentum changes at the shock are purely kinematic, and do not depend on the transport properties. Subdiffusion does change the escape probability at the shock: Kirk et al. (1996) show that the trapping of particles in field line patches increases the escape probability to $\mathcal{P}_{\text {esc }}=6 V_{\mathrm{s}} / r v$, a factor $3 / 2$ larger than in the diffusive case. In the subdiffusive case, the cosmic ray density at the shock is $2 / 3$ of the asymptotic density far downstream, which causes the increased value of $\mathcal{P}_{\text {esc }}$. At low energies, where the subdiffusive limit applies, the spectrum of accelerated particles steepens:

$$
s=1+\frac{\ln \left(1 / \mathcal{P}_{\text {ret }}\right)}{\ln \left\langle p_{n+1} / p_{n}\right\rangle}=\frac{2 r+7}{2(r-1)} .
$$

For a strong shock in an ideal monoatomic gas with $r=4$, this yields $s=2.5$, as opposed to $s=2$ in the diffusive case.

\section{Relativistic Shocks}

There is renewed interest in relativistic shocks with $\Gamma_{\mathrm{s}}=\left(1-\beta_{\mathrm{s}}^{2}\right)^{-1 / 2} \gg 1$ as sources of energetic particles. They have been proposed as the production sites for Ultra-High Energy Cosmic Rays (UHECSs: observed energies 10 ${ }^{18.5}$ $10^{20.5} \mathrm{eV}$ ) (Waxman 1995; Vietri 1995), and must play a role in the generation of the X-ray, optical, and radio afterglows associated with Gamma Ray Bursts (GRBs; see, e.g., Mészáros \& Rees 1997; Sari, Piran, \& Narayan 1998). The outer blast waves of GRBs must have bulk Lorentz factors $\Gamma_{\mathrm{s}} \sim 100-1000$.

Particle acceleration at transrelativistic $\left(\Gamma_{s} \lesssim 10\right)$ shocks has been investigated by a number of authors (e.g., Kirk \& Schneider 1987a, 1987b, 1988) assuming elastic scattering in the up- and downstream frames. These authors treat the scattering with an eigenfunction approach and do not make the standard diffusion approximation of nonrelativistic DSA, which is invalid here.

At ultrarelativistic shocks, with $\Gamma_{\mathrm{s}} \gg 1$, relativistic beaming becomes important. The interaction between particles and the shock is qualitatively different for the first and subsequent shock encounters (Gallant \& Achterberg 1999). At the first encounter, upstream particles are distributed isotropically in the upstream frame. Particles that return upstream due to downstream scattering are boosted to an upstream energy $\sim \Gamma_{\mathrm{s}}^{2}$ times their initial energy (cf. Vietri 1995). Particles re-entering the upstream region are confined to a "loss cone" around the shock normal with opening angle $\theta_{\mathrm{c}} \leq 1 / \Gamma_{\mathrm{s}}$; only these particles can outrun the shock $\left(\boldsymbol{v} \cdot \hat{\boldsymbol{n}}>V_{\mathrm{s}}\right)$. Upstream scattering or deflection is only capable of deflecting a particle out of the loss cone to $\theta \lesssim 2 / \Gamma_{\mathrm{s}}$ before it is recaptured by the shock (Gallant \& Achterberg 1999). This implies that the upstream particle distribution is very anisotropic. The small upstream deflection $\left(\Delta \theta \lesssim 2 / \Gamma_{\mathrm{s}}\right)$ limits the energy gain at subsequent crossing cycles to $\mathcal{E}_{n+1} / \mathcal{E}_{n} \sim 2$. 
Because of the large shock velocity ( $\beta_{\text {sd }} \sim 0.3$ in the downstream frame), the escape probability is high: $\mathcal{P}_{\text {esc }} \approx 0.5$. Simulations (Bednarz \& Ostrowski 1998; Achterberg, Gallant, \& Kirk, in preparation) show that the resulting spectrum is once again a power-law, with a slope $s \sim 2.2-2.3$, depending on the details of the scattering/deflection mechanism.

\section{References}

Achterberg, A. 1979, A\&A, 76, 276

Achterberg, A. 1981, A\&A, 98, 195

Achterberg, A., Blandford, R. D., \& Periwal, V. 1984, A\&A, 132, 97

Achterberg, A., \& Ball, L. 1994, A\&A, 285, 687

Alekseyev, I. I., \& Kroptokin, A. P. 1970, Geomagn. Aeron., 10, 755

Armstrong, T. P., Pesses, M. E., \& Decker, R. B. 1985, in Collisionless Shocks in the Heliosphere: Reviews of Current Research, eds. B. T. Tsurutani \& R. G. Stone (Washington, D. C.: American Geophysical Union), 271

Axford, W. I. 1981, Ann. NY Acad. Sci., 375, 297

Axford, W. I., Leer, E., \& Skadron, G. 1977, Proc. 15 Int. Cosmic Ray Conf. (Budapest: Central Research Institute for Physics) 11, 132

Baade, W., \& Zwicky, F. 1934, Proc. Nat. Acad. Sci., 20, 259

Ball, L., \& Kirk, J. G. 1992, ApJ, 396, L42

Baring, M. G., Ellison, D. C., \& Jones, F. C. 1993, ApJ, 409, 327

Baring, M. G., Ellison, D. C., \& Jones, F. C. 1994, ApJS, 90, 547

Baring, M. G., Ellison, D. C., \& Jones, F. C. 1995, ApJ, 453, 873

Bednarz, J., \& Ostrowski, M. 1998, Phys. Rev. Lett., 80, 3911

Begelman, M. C., Rudak, D., \& Sikora, M. 1990, ApJ, 363, 38

Begelman, M. C., \& Zweibel, E. G. 1994, ApJ, 431, 689

Bell, A. R. 1978, MNRAS, 182, 147

Blandford, R. D., \& Eichler, D. 1987, Phys. Rep., 154, 1

Blandford, R. D., \& Ostriker, J. P. 1978, ApJ, 221, L29

Burn, B. J. 1975, A\&A, 45, 435

Colgate, S. A., \& Johnson, M. H. 1960, Phys. Rev. Lett., 5, 235

de Hoffmann, T., \& Teller, E. 1950, Phys. Rev., 80, 692

Dolginov, A. Z., \& Katz, M. E. 1994, Phys. Rep., 239, 285

Drury, L. O'C. 1983, Rep. Progr. Phys., 46, 963

Drury, L. O'C. 1991, MNRAS, 251, 340

Drury, L. O'C., \& Falle, S. A. E. G. 1986, MNRAS, 223, 353

Drury, L. O'C., \& Völk, H. J. 1981, ApJ, 248, 344

Drury, L. O'C., Völk, H. J., \& Berezhko, E. G. 1995, A\&A, 299, 222

Duffy, P., Drury, L. O'C., \& Völk, H. 1994, A\&A, 291, 613

Duffy, P., Kirk, J. G., Gallant, Y. A., \& Dendy, R. O. 1995, A\&A, 302, L21

Edminston, J. P., Kennel, C. F., \& Eichler, D. 1982, Geophys. Res. Lett., 9, 531 
Eichler, D. 1979, ApJ, 229, 419

Eichler, D. 1984, ApJ, 277, 429

Ellison, D. C., Giacalone, J., Burgess, D., \& Schwartz, S. J. 1993, J. Geophys. Res., 98,21085

Ellison, D. C., Baring, M. G., \& Jones, F. C. 1995, ApJ, 453, 873

Falle, S. A. E. G., \& Giddings, J. R. 1987, MNRAS, 225, 399

Fermi, E. 1949, Phys. Rev., 75, 1169

Forman, M., \& Morfill, G. 1979, Proc. 16th Int. Cosmic Ray Conf. (Tokyo: Institute for Cosmic Ray Research), 5, 328

Gallant, Y. A., \& Achterberg, A. 1999, MNRAS, 305, L6

Hall, D. E., \& Sturrock, P. A. 1967, Phys. Fl., 10, 1593

Hudson, P. D. 1965, MNRAS, 131, 23

Isichenko, M. B. 1991, Plasma Phys. Contr. Fusion, 33, 795

Jokipii, J. R. 1982, ApJ, 255, 716

Jokipii, J. R. 1987, ApJ, 313, 842

Jokipii, J. R., \& Parker, E. N. 1969, ApJ, 155, 777

Jones, F. C., \& Ellison, D. C. 1991, Space Sci. Rev., 58, 259

Kang, H., \& Jones, T. W. 1990, ApJ, 353, 149

Kang, H., \& Jones, T. W. 1995, ApJ, 447, 944

Kazanas, D., \& Ellison, D. C. 1986, ApJ, 304, 178

Kennel, C. F., \& Petchek, H. E. 1966, J. Geophys. Res., 71, 1

Kirk, J. G. 1994, in Plasma Astrophysics, Saas-Fee Advanced Course 24, eds.

A. O. Benz \& T. J-L. Couvoisier (Berlin: Springer), 262

Kirk, J. G., \& Schneider, P. 1987a, ApJ, 315, 425

Kirk, J. G., \& Schneider, P. 1987b, ApJ, 322, 256

Kirk, J. G., \& Schneider, P. 1988, A\&A, 201, 177

Kirk, J. G., Duffy, P., \& Gallant, Y. A. 1996, A\&A, 314, 1010

Krimsky, G. E. 1977, Dokladay Acad. Nauk. SSR, 242, 1306

Koyama, K., Petre, R., Gotthelf, E. V., Hwang, U., Matsuura, M., Ozaki, M., \& Holt, S. S. 1995, Nature, 378, 255

Malkov, M. A., \& Völk, H. J. 1995, A\&A, 300, 605

Malkov, M. A., \& Völk, H. J. 1996, ApJ, 473, 347

Mészáros, P., \& Rees, M. J. 1997, ApJ, 476, 232

Mond, M., \& Drury, L. O'C. 1998, A\&A, 332, 385

Norman, C. A., Melrose, D. B., \& Achterberg, A. 1995, ApJ, 454, 60

Parker, E. N. 1965, Planet. Space Sci., 13, 9

Sari, R., Piran, T., \& Narayan, R. 1998, ApJ, 497, L17

Scholer, M., Kucharek, H., \& Trattner, K. J. 1997, Adv. Space Res., 21, 533

Shatzman, E. 1967, Ann. d'Astr., 26, 234

Sikora, M., Rudak, Kirk, J. G., Begelman, M. C., \& Schneider, P. 1987, ApJ, 341, L33

Skilling, J. 1975a, MNRAS, 172, 557 
Skilling, J. 1975b, MNRAS, 172, 567

Vietri, M. 1995, ApJ, 453, 883

Waxman, A. 1995, Phys. Rev. Lett., 75, 386

Wentzel, D. G. 1974, ARA\&A, 12, 71

Whipple, E. C., Northrop, T. G., \& Birmingham, T. J. 1986, J. Geophys. Res., 91,4149

Zank, G. P., Axford, W. I., \& McKenzie, J. F. 1990, A\&A, 233, 275

Zdziarski, A. A. 1986, ApJ, 305, 45 